\title{
Mantle cell lymphoma presenting as diffuse esophageal, gastric, and duodenal bulb polyposis
}

\section{Papatsakorn Nopjaroonsria, Charoen Mankongpaisarnrung ${ }^{b}$}

Sawanpracharak Hospital, Nakorn, Sawan, Thailand; University of South Dakota, Sanford School of Medicine, Sioux Falls, SD, USA

A 66-year-old male with a history of hypertension presented with a 2-month history of epigastric pain with $4.5-\mathrm{kg}$ involuntary weight loss, nausea with vomiting, but no night sweat. Physical signs included mildly pale conjunctiva without jaundice. There was no lymphadenopathy or hepatosplenomegaly. Esophagogastroduodenoscopy showed diffuse esophageal, gastric, and duodenal bulb polyposis, raising concern for infiltrative malignancy (Fig. 1). Multiple biopsies were obtained. Pathology confirmed the diagnosis of Mantle cell lymphoma (MCL) with positive immunohistochemistry for CD5, CD20, Bcl-2, cyclin D1 and negative immunohistochemistry for $\mathrm{CD} 3, \mathrm{CD} 10, \mathrm{CD} 23$ (Fig. 2). A prompt computed tomography scan of the chest, abdomen and pelvis with contrast showed intrathoracic lymphadenopathy (LAD) with diffuse lobulated enhancing wall thickening of the stomach and pylorus causing severe pyloric canal narrowing and intraabdominal LAD. No evidence of lymphomatous involvement was found on bone marrow biopsy. Unfortunately, the patient died as a result of extensive disease involvement before chemotherapy could be initiated.

The gastrointestinal tract is one of the most common extranodal locations of lymphoma (up to $30 \%$ ) [1-3]. MCL is typically diagnosed in middle-aged men, with a mean age of 63 years [2], and most commonly involves the stomach, followed by the small bowel, and ileocecal valve [1]. MCL rarely involves the esophagus because of the relatively low number of lymphoid cells. The prognosis in MCL is very poor as it usually demonstrates aggressive behavior and is diagnosed at stage 4 in up to $70 \%$ of cases [1-3]. Our report highlights a very interesting endoscopic finding that should be recognized to facilitate prompt clinical management.

\footnotetext{
aSawanpracharak Hospital, Nakorn Sawan, Thailand (Papatsakorn Nopjaroonsri); 'banford Gastroenterology, University of South Dakota, Sanford School of Medicine, Sioux Falls, SD, USA (Charoen Mankongpaisarnrung)
}

Conflict of Interest: None

Correspondence to: Charoen Mankongpaisarnrung, MD, 1205 South Grange Ave, Sioux Falls, SD, 57105, USA,

e-mail: Charoen.Mankongpaisa@usd.edu

Received 23 December 2018; accepted 3 January 2019;

published online 23 January 2019

DOI: https://doi.org/10.20524/aog.2019.0356

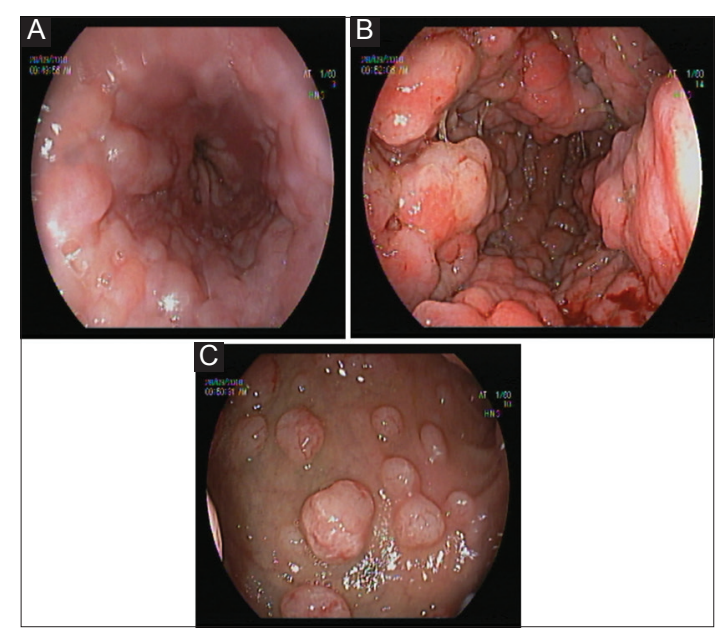

Figure 1 Infiltrative polyposis covering (A) distal esophagus, (B) antrum, causing luminal narrowing, and (C) duodenal bulb
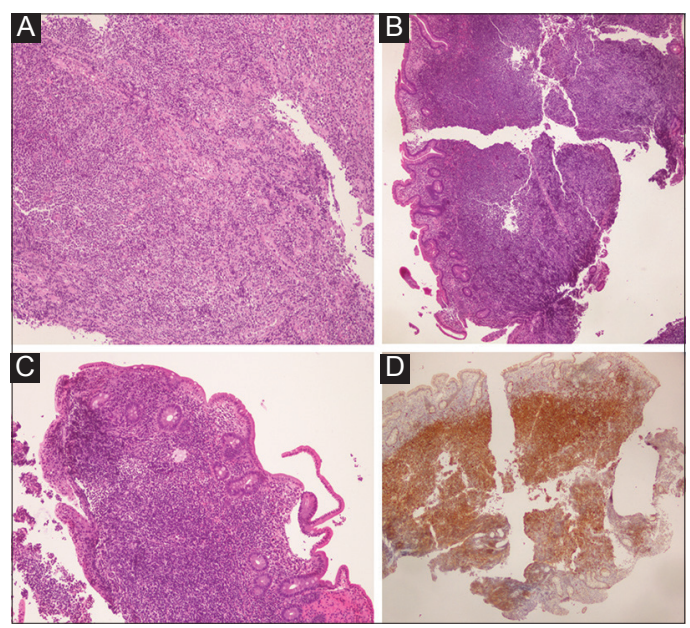

Figure 2 Abnormal lymphomatous cells in (A) esophagus, (B) gastric mucosa, and (C) duodenal bulb. (D) Positive immunohistochemistry of Cyclin D in the duodenum

\section{References}

1. Martins C, Teixeira C, Gamito É, Oliveira AP. Mantle cell lymphoma presenting as multiple lymphomatous polyposis of the gastrointestinal tract. Rev Bras Hematol Hemoter 2017;39:73-76.

2. Lee JA, Taxier MS, Nicely CJ. Electronic clinical challenges and images in GI. Lymphomatous polyposis. Gastroenterology 2008;135:e1-e3.

3. Michopoulos S, PetrakiK, Matsouka C, KastritisE, Chrysanthopoulou H, Dimopoulos MA. Mantle-cell lymphoma (multiple lymphomatous polyposis) of the entire GI tract. J Clin Oncol 2008;26:1555-1557. 